\begin{tabular}{|c|c|c|}
\hline \multirow{3}{*}{$\begin{array}{r}\text { Case Reports in } \\
\text { Gastroenterology }\end{array}$} & \multirow{2}{*}{\multicolumn{2}{|c|}{ Case Rep Gastroenterol 2013;7:433-437 }} \\
\hline & & \\
\hline & $\begin{array}{l}\text { DOI: 10.1159/000355881 } \\
\text { Published online: October 9, } 2013\end{array}$ & $\begin{array}{l}\text { ○ } 2013 \text { S. Karger AG, Basel } \\
\text { 1662-0631/13/0073-0433 } \$ 38.00 / 0 \\
\text { www.karger.com/crg }\end{array}$ \\
\hline & \multicolumn{2}{|c|}{$\begin{array}{l}\text { This is an Open Access article licensed under the terms of the Creative Commons } \\
\text { Attribution-NonCommercial } 3.0 \text { Unported license (CC BY-NC) (www.karger.com/OA } \\
\text { license), applicable to the online version of the article only. Distribution permitted for non } \\
\text { commercial purposes only. }\end{array}$} \\
\hline
\end{tabular}

\title{
A Case of Adenocarcinoma of the Duodenum Arising from Brunner's Gland
}

\author{
Keiko Kamei Takeo Yasuda Takuya Nakai Yoshifumi Takeyama \\ Department of Surgery, Kinki University Faculty of Medicine, Osakasayama, Japan
}

\section{Key Words}

Duodenal carcinoma $\cdot$ Brunner's gland

\begin{abstract}
We report a rare case of adenocarcinoma of the duodenum arising from Brunner's gland. A 70-year-old man with a history of hypertension was referred to us with the complaint of abdominal discomfort. Upper gastrointestinal endoscopy revealed an irregular elevated mucosa and a submucosal tumor with delle in the duodenal bulb. Biopsy specimens revealed adenocarcinoma (the former) and hyperplasia (the latter). We could not agree with the patient about performing pancreaticoduodenectomy, so under the diagnosis of primary duodenal carcinoma, we performed resection of the bulbus and the antrum. Pathological examination showed that one of the tumors was consistent with normal Brunner's glands, Brunner's gland hyperplasia and adenocarcinoma arising from Brunner's glands. The patient's postoperative course was good, but 15 months after, he developed lymph node recurrence at the site of the pancreas head. Under the diagnosis of lymph node metastasis of duodenal cancer, we performed pancreaticoduodenectomy this time. Pathological examination confirmed our preoperative diagnosis. The patient remained well after the surgery for 2 years.
\end{abstract}

\section{Introduction}

Except for carcinoma of the ampulla of Vater, primary malignant tumors of the duodenum are rare. It was reported that primary adenocarcinoma of the duodenum accounted for $0.3 \%$ of all carcinomas of the gastrointestinal tract. Brunner's glands exist from the pylorus to the jejunum [1], mainly in the first and the second section of the duodenum amongst men aged 40-60 years [2]. In recent years, due to the advances of modalities for investigating the

Takeo Yasuda

Department of Surgery, Kinki University Faculty of Medicine

377-2 Onohigashi

Osakasayama 589-8511 (Japan)

E-Mail yasudatk@surg.med.kindai.ac.jp 
Kamei et al.: A Case of Adenocarcinoma of the Duodenum Arising from Brunner's Gland

upper gastrointestinal tract, primary carcinoma of the duodenum has increased, but adenocarcinoma arising from Brunner's gland is still very rare. In this paper, we report a case of adenocarcinoma of the duodenum arising from Brunner's gland, with a review of the literature.

\section{Case Report}

A 70-year-old man was referred to our hospital for investigation of his abdominal discomfort. He had been followed with a diagnosis of diabetes mellitus for 45 years. On admission, physical examination showed no abnormalities, and all laboratory data were normal, except for an elevation in CEA and CA19-9. His CEA level was $128 \mathrm{ng} / \mathrm{ml}$ (normal 0-5 ng/ml) and his CA19-9 was $326 \mathrm{U} / \mathrm{ml}$ (normal 0-37 U/ml). Upper gastrointestinal endoscopy revealed an irregular elevated mucosa (fig. 1a) and a submucosal tumor with delle in the duodenal bulb (fig. 1b). Biopsy specimens revealed adenocarcinoma (the former) and hyperplasia (the latter). Abdominal computed tomography confirmed the presence of a mass originating in the submucosal layer of the duodenal bulb.

Under the diagnosis of adenocarcinoma of the duodenum, we proposed to perform pancreaticoduodenectomy, but the patient did not consent. Thus, partial resection of the bulbus and the antrum was performed instead. At laparotomy, we saw that the tumor had not invaded the serosa and that lymph node swelling was limited, so we dissected the lymph nodes, including No. 6, 8a, 8p, 12a and 16b1. The resected specimen showed two tumor types, in accordance with the preoperative findings (fig. 2). Pathological examination of the oral side of the tumor showed that it was consistent with normal Brunner's glands (fig. 3a, black arrows), Brunner's gland hyperplasia (fig. 3a, white arrow) and adenocarcinoma supposed to arise from Brunner's glands (fig. 3a, black arrowheads). The anal side of the tumor also revealed Brunner's gland hyperplasia and adenoma supposed to arise from Brunner's glands (fig. 3c). Immunohistochemical staining revealed that the site of normal and hyperplasia gland was positive for MUC5AC (data not shown) and MUC6 (fig. 3b, d), but the site of adenoma and adenocarcinoma was negative. Lymph node metastasis was positive for No. 6 and 8a. The patient's postoperative course was good, but adjuvant chemotherapy was not done because the he refused it. Fifteen months after the operation, laboratory data showed high levels of CEA and CA19-9 $(45 \mathrm{ng} / \mathrm{ml}$ and $237 \mathrm{U} / \mathrm{ml})$. Positron emission tomography and computed tomography revealed a tumor located near the head of the pancreas. Under the diagnosis of lymph node metastasis of duodenal cancer, we did pancreaticoduodenectomy. Pathological examination confirmed our preoperative diagnosis. The patient received adjuvant chemotherapy with $S-1$ and remained well after the surgery for 2 years.

\section{Discussion}

Carcinoma of the duodenum arising from Brunner's gland was first reported in 1894 by Pic [3]. Nowadays increasing reports about this disease reveal its clinicopathological feature. Brunner's glands exist mainly in the first and second section of the duodenum. Brunner's glands are mucus-secreting acinar gland located in the deep mucosa or submucosa of the intestine [4, 5], so adenocarcinoma arising from Brunner's gland looks like a submucosal tumor or polypoid lesion in the early stage [6]. As it progresses, tumors look like Borrmann 
Kamei et al.: A Case of Adenocarcinoma of the Duodenum Arising from Brunner's Gland

type 2. In our case, the anal side tumor revealed typical submucosal tumor, and the oral side tumor looked like Borrmann type 2, suggesting the natural history of Brunner's tumor.

The diagnosis was made by histological examination, but it is thought to be difficult to ascertain its cellular origin by H\&E staining alone. Nowadays, the utility of immunohistochemical staining with MUC6 is demonstrated. The MUC6 gene is thought to produce the class III mucin, which is specific for Brunner's glands, pyloric glands and mucus neck cells of the stomach. In our case, immunohistochemical staining with MUC6 of hyperplasia of Brunner's gland was positive, but the adenoma and adenocarcinoma components were negative for MUC6. MUC5AC, which is the marker for gastric foveolar-type mucin, was also positive in the component of hyperplasia, but negative in adenoma and adenocarcinoma. These changes in immunohistochemical staining showed that Brunner's glands lost its nature with tumor progression.

The treatment strategy of adenocarcinoma of Brunner's gland is still controversial. Like in other carcinoma of the duodenum, the method of operation depends on its location and on tumor invasion depth. Concerning duodenal adenocarcinoma, Kerremans et al. [7] reported that Whipple resection should not be performed in the presence of regional lymph node invasion, because if regional lymph node invasion existed, there was no differences in mean survival time between the Whipple resection group and the palliative operation group. Kaklamanos et al. [8] also reported that segmental duodenal resection for patients with duodenal adenocarcinoma is associated with acceptable long-term survival and that clearance of lymph nodes and outcome are comparable to pancreaticoduodenectomy. Recently, Gold et al. [9] reported that lymph node status was a significant prognostic variable and that the use of pancreaticoduodenectomy was not a significant prognostic variable. However, concerning our case, if pancreaticoduodenectomy had been done straight away, complete remission might have been achieved with one operation.

The prognosis of duodenal cancer is poor. Hung et al. [10] reported a 5-year survival rate in primary duodenal adenocarcinoma of $7.9 \%$. Bridge et al. [11] reported the importance of having metastases to the lymph nodes.

\section{Conclusion}

We report a case of adenocarcinoma of the duodenum arising from Brunner's gland. It is rare but should be treated carefully to obtain a complete cure.

\section{Disclosure Statement}

There are no conflicts of interest.

\section{References}

1 Kaplan EL, Dyson WL, Fitts WT: Hyperplasia of Brunner's glands of the duodenum. Surg Gynecol Obstet 1968;127:371-375.

2 Sande-Lemos Azcue MP, Shaoul R, Cutz E, Sherman PM: Nodular duodenum after pediatric renal transplant due to Brunner's gland hyperplasia. J Pediatr Gastroenterol Nutr 1998;27:452-456.

3 Pic A: Contribution à l'étude du cancer primitif du duodénum. Rev Med 1894;14:1081-1101.

-4 Parkhomenko YG, Grunko VA, Kozlov AI: Signet-cell carcinoma of Brunner's glands. Arkh Patol 1990;55: $55-58$.

5 Leeson TS, Leeson CR: The fine structure of Brunner's glands in man. J Anat 1968;103:263-276. 
Kamei et al.: A Case of Adenocarcinoma of the Duodenum Arising from Brunner's Gland

6 Shinohara T, Terasaki M, Kuno T, Okamoto Y, Sakamoto E, Kamiya S, Kobayashi S, Asaba Y, Hoshi S: A case of cancer in adenoma arising in Brunner's glands. Jpn J Gastroenterol Surg 2000;33:338-341.

7 Kerremans RP, Lerut J, Penninckx FM: Primary malignant duodenal tumors. Ann Surg 1979;190:179-182.

8 Kaklamanos IG, Bathe OF, Franceschi D, Camarda C, Levi J, Livingstone AS: Extent of resection in the management of duodenal adenocarcinoma. Am J Surg 2000;179:37-41.

-9 Gold JS, Tang LH, Gönen M, Coit DG, Brennan MF, Allen PJ: Utility of a prognostic nomogram designed for gastric cancer in predicting outcome of patients with R0 resected duodenal adenocarcinoma. Ann Surg Oncol 2007;14:3159-3167.

10 Hung FC, Kuo CM, Chuah SK, Kuo CH, Chen YS, Lu SN, Chang Chien CS: Clinical analysis of primary duodenal adenocarcinoma: an 11-year experience. J Gastroenterol Hepatol 2007;22:724-728.

11 Bridge MF, Perzin KH: Primary adenocarcinoma of the jejunum and ileum. A clinicopathologic study. Cancer 1975;36:1876-1887.
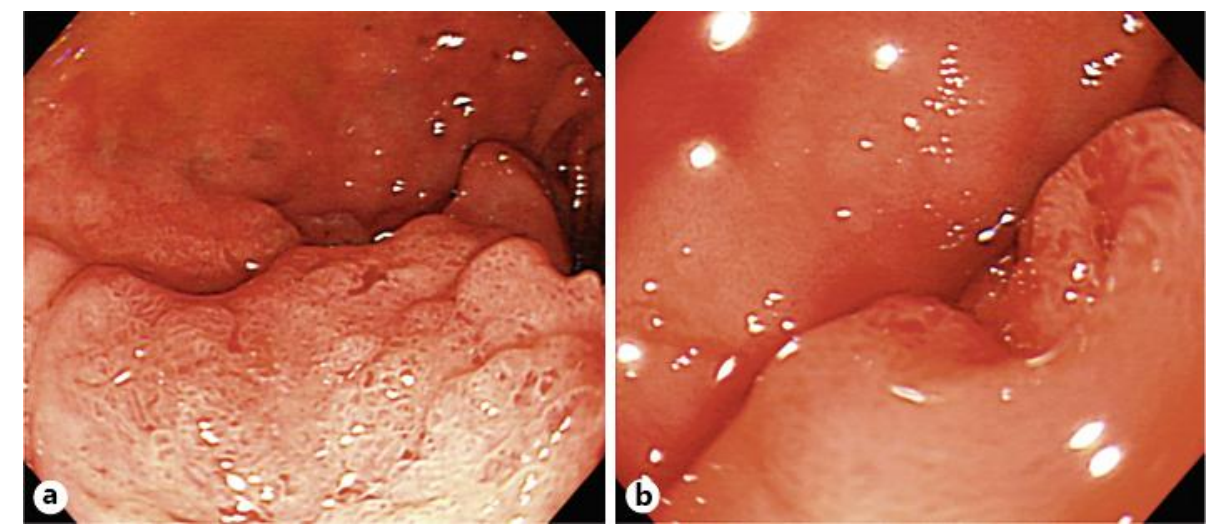

Fig. 1. a Upper gastrointestinal endoscopy revealed an irregular elevated mucosa in the duodenal bulb. b The other tumor looked like a submucosal tumor with delle.

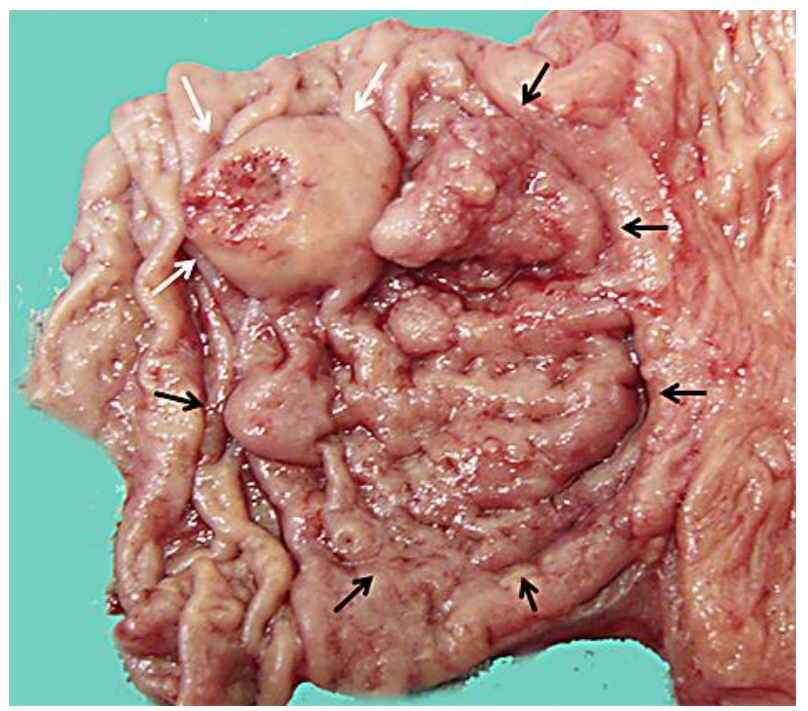

Fig. 2. The resected specimen shows Borrmann type 2 tumor (black arrows) and submucosal tumor (white arrows). 
Kamei et al.: A Case of Adenocarcinoma of the Duodenum Arising from Brunner's Gland
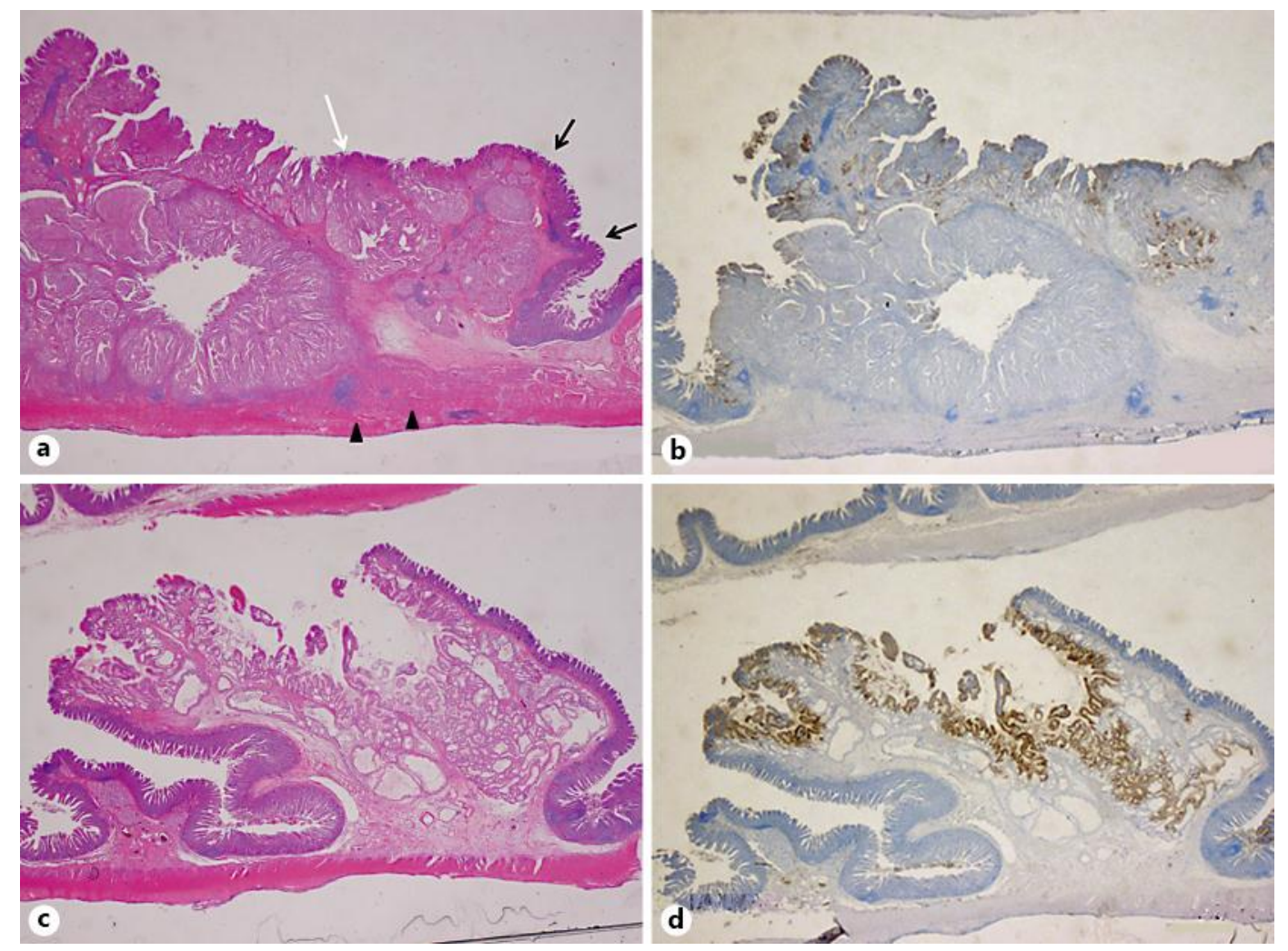

Fig. 3. a Pathological examination of the oral side tumor showed that it was consistent with normal Brunner's glands (black arrows), Brunner's gland hyperplasia (white arrow) and adenocarcinoma supposed to arise from Brunner's glands (black arrowheads) (H\&E, ×40). b Immunohistochemical staining revealed that the sites of normal and hyperplasia glands were positive for MUC6, but that the site of adenocarcinoma was negative $(\times 40)$. c Pathological examination of the anal side tumor reveals normal Brunner's glands and adenoma supposed to arise from Brunner's glands (H\&E, $\times 40$ ). d Immunohistochemical staining revealed that the sites of hyperplasia glands were positive for MUC6, but that the site of adenoma was negative $(\times 40)$. 